\title{
Significance and Clinical Effect of Anteromedial Support Plate Assisted Reduction and Fixation in The Treatment of Complex Proximal Humeral Fractures
}

\author{
XiaoTian Ma ( $\nabla$ mxt18021566700@163.com ) \\ Affiliated Hospital of Jiangnan University https://orcid.org/0000-0002-3871-1946 \\ WeiYa Zhang \\ yancheng no. 1 people's hospital \\ Zheng Xu \\ zhangjiagang no.1 people's hospital
}

\section{Research Article}

Keywords: proximal humeral fracture, medial support, internodal groove, locking plate

Posted Date: October 27th, 2021

DOl: https://doi.org/10.21203/rs.3.rs-989301/v1

License: (c) (1) This work is licensed under a Creative Commons Attribution 4.0 International License.

Read Full License 


\section{Abstract \\ Background}

There are more and more complex consolidated proximal fractures, and the postoperative effect is not very satisfactory. Therefore, we propose a surgical method and evaluate its effect.

\section{Method}

A total of 77 patients with complicated proximal humeral fractures who underwent surgical treatment in the Affiliated Hospital of Nantong University from June 2015 to January 2019 were collected. Among them, the internodal groove medial support plate assisted reduction and fixation technique and the lateral locking plate were used. A total of 15 cases of complicated proximal humeral fractures were used as the study group, and 62 patients with the same type of proximal humeral fractures treated with traditional reduction methods and internal fixation with lateral locking plates during the same period were used as the control group.The operation time of the two groups; intraoperative blood loss; number of effective intraoperative fluoroscopy; postoperative humeral neck shaft angle loss after reduction; postoperative shoulder joint Constant-Murley score; upper limb function DASH score and postoperative complications were retrospectively reviewed comparative analysis.

\section{Results}

The number of effective intraoperative fluoroscopy was (6.18 1.869$)$ times in the control group, more than $(3.93 \pm 1.387)$ times in the study group, and the difference between the two was statistically significant $(P<0.05)$; The operation time of the study group was longer than that of the control group, and the intraoperative blood loss of the study group was more than that of the control group, but the difference between the two was not statistically significant $(P>0.05)$.In the first $1,3,6$, and 12 months after surgery, in terms of imaging measurement, the loss angle after reduction of the humeral neck shaft angle in the control group and the study group increased with the increase in postoperative time. There was no significant difference in the lost angle after reduction of the humeral neck shaft angle between the two groups at month and 3 months $(P>0.05)$. At the 6th and 12th months after surgery, the lost angle after reduction of the humeral neck shaft angle in the study group was smaller than that in the control group, and the difference between the two groups was statistically significant $(P<0.05)$. For the recovery of shoulder joint function after surgery, the Constant-Murley score of the shoulder joint and the DASH score of upper limb function of the control group and the study group increased with the increase of postoperative time, while the Constant score and DASH score of the two groups were 1 after surgery. There was no significant difference at months, 3 months, 6 months and 12 months $(P>0.05)$.

\section{Conclusion}


The anteromedial support plate assisted reduction of the internodal groove can be used as a single locking plate for the treatment of complex proximal humeral fractures with medial column instability. It is an option when reduction is difficult or it is difficult to maintain stable reduction, which improves the quality of reduction and reduces surgery. Loss of posterior fracture reduction.

\section{Introduction}

The shoulder joint is the joint with the largest range of motion in the human body. Although a certain degree of deformity will not cause obvious dysfunction, at present, orthopedic surgeons still face great difficulties in the treatment of complex proximal humeral fractures [1].At present, locking plate fixation has become an effective treatment for complex proximal humeral fractures [3], but when using lateral locking plates to treat proximal humeral fractures with medial column injury, there is still a high postoperative complication. Rate, up to $25 \%$ [4].Due to the lack of effective reduction and the maintenance of stability after reduction during the operation, and it is difficult to accurately fix the plate to the anterior edge of the humeral shaft, these factors lead to the possibility of rotation or angular deformation after the use of a single locking plate to reduce the fracture, which will eventually lead to fractures. The prognosis is poor [5]. Therefore, how to ensure the stability of the medial column of the humerus to ensure the quality of surgical reduction is a difficult point in the treatment of proximal humeral fractures. We have proposed a treatment plan for the treatment of complex proximal humeral fractures with the aid of reduction and fixation through the anteromedial support plate of the intertubular groove. This plan has certain feasibility. The anteromedial support of the intertubercular groove can better maintain the stability of the media during the operation, so that the stress distribution on the medial proximal humerus after fracture is as close as possible to the normal stress distribution on the medial humerus, ensuring maximum recovery of the humeral neck The dry angle allows fracture reduction to be carried out in the best way, which will help fracture healing and reduce the occurrence of poor fracture union and fracture complications [6].This article aims to investigate the clinical application of this technique by reviewing the short- and medium-term clinical efficacy and radiological results of the treatment of complex proximal humeral fractures with the anteromedial support plate assisted reduction and fixation of the transtubercular interstitial sulcus and the results of simple locking plate fixation.

\section{Clinical research}

objectnThe subjects were 77 patients with complex proximal humeral fractures treated with lateral locking plate fixation and medial support plate plus lateral locking plate fixation in the Department of orthopaedics of the Affiliated Hospital of Nantong University from June 2015 to January 2019 (Neer type III or Type IV).Among them, 15 cases of complex proximal humeral fractures treated with medial support plate assisted reduction and lateral locking plate were used as the study group. There were 9 females and 6 males, aged 58 to 83 years old, with an average of $(66.5 \pm 12.8)$ years old.A total of 62 cases of the same type of proximal humeral fractures treated with traditional reduction methods and internal fixation 
with lateral locking plates during the same period were used as the control group. There were 41 females and 21 males, aged 53 to 81 years old, with an average of $(59.2 \pm 15.5)$ years old.

\section{Method}

Inclusion criteria: هComplicated proximal humeral fractures (Neer classification of three-part and four-part fractures); $₫$ Unilateral injury; $\mathbb{\nabla}$ The causes of injuries in all patients are similar, mostly caused by indirect

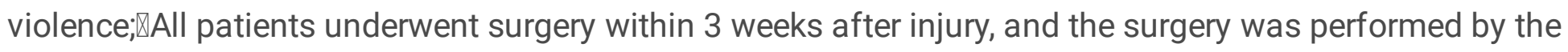

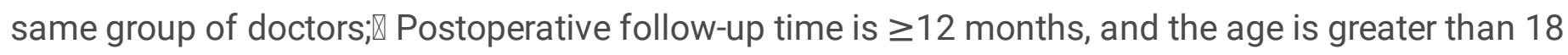
years.

Exclusion criteria: $₫$ Complicated with nerve injury or neuromuscular disease; $\mathbb{0}$ pen fracture; $₫$ Multiple

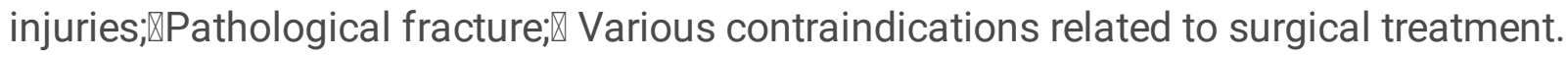

\section{Evaluation standard:}

According to the operation records and anesthesia records of the two groups of patients, the number of intraoperative fluoroscopy, operation time and intraoperative blood loss were collected and compared.The shoulder joint function Constant score and upper limb function DASH score were performed at $1,3,6$, and 12 months after the operation. X-rays were re-examined several times, and the lost angle of the humeral neck shaft angle of the two groups was recorded and calculated [7] .Corresponding data were compared according to the measurement and calculation results of the lost angle of the humeral neck shaft angle after reduction and the changes in the Constant score of the shoulder joint function and the DASH score of the upper extremity function obtained during the follow-up at 1, 3, 6, and 12 months after the operation.

\section{Preoperative preparation:}

\All patients undergo routine examinations after admission to assess their cardiopulmonary function; $₫$ Further imaging examinations, including standard anterolateral $x$-rays and CT3D imaging of the affected shoulder; खRoutine analgesic and swelling treatment.खElderly patients with chronic diseases such as hypertension and diabetes should control their complications.

\section{Surgical methods:}

All patients underwent intravenous compound anesthesia. After successful anesthesia, they were in supine position or beach chair position. Take the deltoid-pectoralis major space approach, pull the cephalic vein to the inside for protection, and fully expose the fractured end.The humeral head and shaft were reduced by traction and prying, and the rotator cuff attached to the large tubercle or the small tubercle was sutured with No. 5 Ethbon thread for traction, and the large and small tubercle bone pieces were reset.Postoperative c-arm fluoroscopy to monitor the effect of reduction and fixation.According to the different reduction and fixation methods during the operation, they were divided into a simple lateral plate fixation group (control group) and a medial support plate assisted reduction group (study group). 
Control group:After reduction, several Kirschner wires are used for temporary fixation. After the reduction is satisfactory, the Kirschner wires that obstruct the plate fixation should be removed, and the lateral proximal humerus locking compression plate should be used for fixation.

Study group:First reset the medial bone block and temporarily fix it with several Kirschner wires.Place a pre-bent $1 / 3$ tube-shaped steel plate on the inner side of the proximal humerus, that is, the anterior and inner side of the biceps longiceps tendon groove.The proximal 1-2 cortical screws are fixed to the humeral head, and the distal 2-3 cortical screws are fixed to the humeral shaft.Maintain the stability of the head and neck stem, Then the large and small tubercle bone blocks were reset, the Kirschner wires that obstructed the plate fixation were removed, and the proximal humerus locking compression plate was used to fix the outside.

\section{Postoperative treatment:}

After the operation, the conventional triangle sling was suspended and fixed for 4-6 weeks,Passive forward flexion can be started after 1 week, abduction and internal and external rotation can be increased after 6 weeks, and active muscle training can be started after 12 weeks.X-ray examinations were performed at 1, 3, 6 and 12 months after surgery. The Constant score of shoulder joint function and the DASH score of upper limb function were performed at 1, 3, 6 and 12 months after surgery.

\section{Record postoperative complications and functional evaluation indicators:}

Record the postoperative and follow-up review, evaluate the relevant functional scores,Ask a physician with rich experience in imaging to take x-rays, using the forearm in a neutral position, and then take a front and side view of the shoulder joint to ensure that the forearm is not in an internal or external rotation position.In order to understand whether there is fracture reduction loss, bone healing, and the same person repeats the measurement of the relevant angle 3-5 times, then calculates and compares, and records the relevant complications.

\section{Statistical Analysis:}

The IBM SPSS22.0 software was selected for statistical analysis and processing. The recorded data was expressed by $x \pm s$, the count data such as the number of cases in the general patient data were used by the $\chi 2$ test, and the independent sample t test was used for the numerical data between the two groups. $\mathrm{P}<0.05$ was considered as statistically significant.

\section{Results}

Basic situation:A total of 77 eligible patients were enrolled in this study, and they were all followed up effectively. The basic conditions of the two groups are shown in Table 1.There was no statistically significant difference between the two groups of patients in terms of gender, age, side of injury, Neer classification, and time from injury to operation $(p>0.05)$. The clinical data of the two groups were comparable. 
Perioperative situation:The perioperative data of the two groups of patients are shown in Table 2.The operation time of the study group was longer than that of the control group, and the intraoperative blood loss of the study group was more than that of the control group, but the difference between the two was not statistically significant $(P>0.05)$. The number of intraoperative fluoroscopy in the control group was more than that of the study group. The difference was statistically significant $(P<0.05)$.

Follow-up results:All 77 patients were followed up for at least 12 months, with an average follow-up time of (14.05 \pm 1.72$)$ months. Table 3 shows in detail the Constant scores and comparisons of the shoulder joints at 1, 3, 6, and 12 months after surgery, and Table 4 shows the DASH scores and comparisons of the two groups.At 1, 3, 6, and 12 months after surgery, the Constant scores and DASH scores of the two groups of patients were similar, and there was no statistical difference $(P>0.05)$. Although the two groups of patients recovered slowly in the early stage and had obvious pain symptoms, they were The overall functional recovery was better in the late course of the disease.

Radiological measurement:The measurement and calculation results of the lost angle of the humeral neck shaft angle after reduction are shown in Table 5.There was no statistically significant difference in the loss of the humeral neck shaft angle between the two groups at the first and third months $(P<0.05)$, while at the sixth and 12th months after the operation, the study group's humeral neck shaft angle was reset. The posterior loss angle was smaller than that of the control group, and the difference was statistically significant $(P<0.05)$.

Postoperative complications:One case of incision fat liquefaction occurred in the control group after the operation (improved after active dressing change, the incision healed). One case of skin paresthesia in the deltoid region occurred in the study group after surgery. Considering that the axillary nerve was stretched during the operation, it returned to normal 5 days after the operation. There were no complications such as late humeral head necrosis, loosening of internal fixation, screw breakage, delayed union, nonunion, etc. in both groups.

\section{Discussion}

Proximal humeral fractures are currently a common fracture [8-9], and lateral locking plate fixation is currently the most widely used surgical method for the treatment of proximal humeral fractures [10].However, as time goes by, more and more scholars have found that the postoperative complications of the treatment of complex proximal humeral fractures with lateral locking plates are still high, such as: humeral head varus deformity, fracture displacement, internal Loose fixation, broken screws, etc. [11].The anteromedial support plate assisted reduction technique used in this study has certain advantages in maintaining the reduction of proximal humeral fractures and reducing postoperative complications. The anterior medial plate of the intertubular groove can provide an effective medial stress dispersion for the treatment of proximal humeral fractures, so that the humeral head can obtain effective biological support, thereby increasing the stability of fracture reduction and reducing the loss of fracture reduction.According to the study of Wu JW et al. [12], patients with medial support are better than 
patients without medial support in terms of humeral head height loss, indicating that restoring medial support can maintain the reduction effect and enhance the stability of reduction.Karppinger et al. [13] proved through biomechanical studies that those with support on the inner side of the proximal humerus can increase the axial load by nearly 10 times compared with those without support. It also explains the importance of medial support for maintaining fracture reduction.In addition, this research has improved the traditional double steel plate technology. The traditional double steel plate technology is to reset first, and then place the double steel plate for fixation. In this research, the support plate is placed on the anterior and medial side of the internodal groove first, and then depends on the stable medial support. Perform reduction and fixation, which reduces the difficulty of fracture reduction.

The anterior and posterior humeral arteries are the main blood supply source of the proximal humerus, and the survival of the ascending branch of the anterior humeral artery after the fracture directly affects the occurrence of avascular necrosis of the humeral head [14].Therefore, we need to be especially careful when performing medial reduction and fixation of proximal humeral fractures to protect the medial soft tissues and important blood vessels and nerves. The traditional double plate fixation technique is to place the medial support plate on the inner side of the biceps long head tendon groove. Excessive dissection will inevitably destroy a part of the medial blood supply, and we place the medial plate on the long biceps brachii. The anterior and inner side of the cephalic tendon groove only needs to peel off the partial stop of the pectoralis major muscle, which can better protect the blood supply of the humeral head.

In this study, based on the original lateral locking plate, a pre-curved $1 / 3$ tube-shaped plate was added to the inner side of the proximal humerus during the operation to assist the reduction of complex proximal humeral fractures and enhance the stability of the fracture reduction. At the same time observe its clinical efficacy.In this study, although the surgical procedure required to place two plates in the study group was more complicated than that in the control group, the study group placed a support plate on the medial side to restore the stability of the medial column, simplify the operation, and reduce the loss of position after bone block reduction. It can reduce the number of effective intraoperative fluoroscopy, and complete the reduction and fixation of fractures with better and higher quality. Therefore, there was no statistical difference in the length of operation and the amount of bleeding between the two groups. Park SG et al. [15] also demonstrated this view.At the same time, this also reduces the radiation exposure of the surgeon and the patient during the operation. With the continuous improvement of surgical skills and proficiency, the operation time and blood loss of the use of internal and external double plate treatments will also be reduced.Comparing the loss angle of the humeral neck shaft angle of the two groups after reduction, although there was no significant difference between the two at the first and third months, at the sixth and 12th months, the data of the control group showed higher data and statistics Learn the difference.This shows that the study group has a greater advantage in maintaining the long-term stability of complex proximal humeral fractures after reduction and fixation than the control group. It can also be further inferred that the study group's complications such as humeral head deformity will be less than the control group.Theopold J[16] and others have also reached a similar conclusion.According to Liu ZD [17] and others, when the humeral neck shaft angle is less than $120^{\circ}$ after reduction, it will have an impact on the recovery of shoulder joint function. Therefore, we regard the neck-stem angle $<120^{\circ}$ after reduction as 
meaningful or as the loss of neck-stem angle reduction. In this study, the loss of reduction rate in the control group reached $14.5 \%$, while in the study group, there was no obvious loss of reduction. This further shows that the research group has certain advantages in maintaining stability after reset.Regarding the shoulder joint Constant score and the upper limb function DASH score, although the study group's reexamination scores at each time point were higher than those of the control group, the difference between the two was not statistically significant. The shoulder joint function of the two groups of patients was All had a relatively good recovery after the operation.The possible reason for the small difference in functional scores between the two groups is that we conducted a careful evaluation before the operation and selected the corresponding surgical methods according to different situations, so that the patients can get good treatment.Although there was a difference in the angle loss of the neck-stem angle between the two groups, there was no loss of more than $20^{\circ}$, so there was no significant difference in the recovery of shoulder joint function between the two groups.For complex proximal humeral fractures, especially fractures with medial column injuries, if the support of the medial column cannot be effectively restored during the operation, it will inevitably increase the secondary varus collapse, loss of reduction and final fixation failure of the humeral head due to lack of support. The possibility [18].In this case, only one lateral steel plate cannot effectively maintain the stability of the fracture. Therefore, it is often necessary to use the medial steel plate for the necessary auxiliary support to rebuild the stability of the medial column, and cooperate with the lateral locking plate to complete the reduction and fixation of the fracture[19].The main reason for the use of double plate fixation is that the use of a single lateral locking plate cannot recover and maintain the support of the medial metaphysis to the humeral head due to various reasons during the operation. Therefore, this technology cannot be used as a commonly used clinical fixation technology, and its application indications should be strictly controlled: 1 . The medial metaphysis is damaged, and the bone quality is poor, which is expected to be difficult to restore and fix.2. It is easy to shift after reset and fixation, and the internal fixation fails.3. After reduction and fixation, humeral head varus collapse or head and neck separation is likely to occur.In addition, we recommend that a lot of study and observation should be done before using this technique to prevent invalid fixation due to lack of surgical skills and experience.

In this study, there was 1 case of incision fat liquefaction in the control group.The patient is a 67-year-old obese elderly woman,Surgery was performed on the 10th day after injury,The dressing was found to be moist on the 7th day after surgery,Pressing the incision has yellow exudate, the incision has no redness and swelling, and the skin temperature is not high. It is considered that the fat is liquefied.Our analysis may be due to the thick subcutaneous fat of the patient, the high temperature generated during the operation of the electrosurgical knife that damaged the adipose tissue, and the excessive stretching of the adipose tissue during the fracture reduction. Therefore, for obese patients, it is especially necessary to pay attention to the gentle operation during the operation and avoid excessive use of electric knife to cut fat tissue.One case of axillary nerve palsy occurred in the study group. Considering that it was caused by excessive pulling of the upper extremity in the external rotation position for a long time during the process of fracture reduction and plate placement.During the operation, it is necessary to pay attention to the 
gentle reduction movement, improve the surgical technique, and strengthen the protection of the axillary nerve.

This study has several limitations. First of all, this article is a retrospective study based on the results of follow-up, so the differences in observations may bias the study.Second, the sample size of this study is small, the follow-up time is short, and the sample statistics need to be expanded for long-term research.

\section{Conclusion}

For complex proximal humeral fractures, especially fractures with unstable medial column,The anteromedial support-assisted reduction of the internodal sulcus plus lateral locking plate technology can effectively compensate for the limitations of the unilateral locking plate in the treatment of this type of fracture through the strong fixation of the medial support and the lateral locking plate.That is, a series of complications such as the difficulty of reduction and instability due to the lack of stability of the medial column, and the varus deformity of the humeral head caused by the loss of reduction in the later period [20].The anteromedial auxiliary plate technology of the internodal groove helps to improve the quality of reduction and reduce the loss of the cervical shaft angle after fracture reduction, thereby reducing the occurrence of a series of complications such as humeral head varus deformity caused by the loss of reduction in the later stage of treatment. The clinician provided an alternative surgical plan.

\section{Declarations}

We thank this magazine for publishing this article

\section{Availability of data and materials}

Please contact author for data requests.

\section{Funding}

Not applicable

\section{Author information}

\section{Author note}

XiaoTian Ma is the first author,WeiYa zhang and Zheng Xu are co-first authors, the two authors contributed to this article equally.

\section{Affiliations}

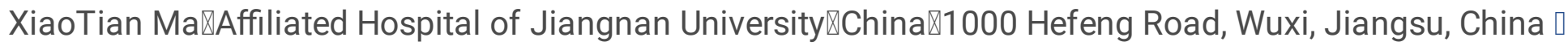
mxt18021566700@163.com 


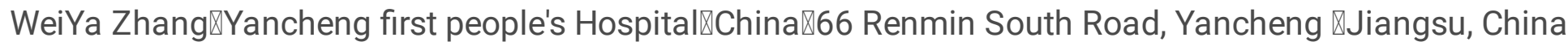
ه1184259954@qq.com

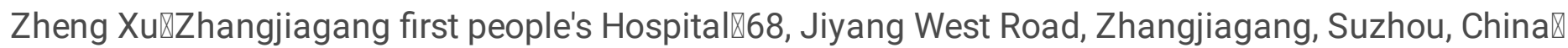
xuzhengny@sina.cn

\section{Contributions}

XTM and WYZ: applied for ethical and registered this study, drafted the work and revised it critically for important intellectual content; ZX : collected date, analyzed and interpreted data for the work;XTM: participated in the collected date and final approval of the version to be published;XTM: contributions to the conception and design of the work, and revised the manuscript. All authors read and approved the final manuscript.

\section{Authors' information}

The work was performed in Department of Affiliated Hospital of Jiangnan University $\mathbb{}$ China.

\section{Corresponding author}

Not applicable

\section{Ethics declarations}

\section{Ethics approval and consent to participate}

The hospital's institutional review board approved the study. Written informed consent was deemed unnecessary by the hospital's institutional review board.

\section{Consent for publication}

No conflict of interest exits in the submission of this manuscript, and manuscript is approved by all authors for publication.

\section{Competing interests}

The authors declare that they have no competing interests.

\section{Publisher's Note}

Springer Nature remains neutral with regard to jurisdictional claims in published maps and institutional affiliations.

\section{Rights and permissions}


Open Access This article is licensed under a Creative Commons Attribution 4.0 International License, which permits use, sharing, adaptation, distribution and reproduction in any medium or format, as long as you give appropriate credit to the original author(s) and the source, provide a link to the Creative Commons licence, and indicate if changes were made. The images or other third party material in this article are included in the article's Creative Commons licence, unless indicated otherwise in a credit line to the material. If material is not included in the article's Creative Commons licence and your intended use is not permitted by statutory regulation or exceeds the permitted use, you will need to obtain permission directly from the copyright holder. To view a copy of this licence, visit http://creativecommons.org/licenses/by/4.0/. The Creative Commons Public Domain Dedication waiver (http://creativecommons.org/publicdomain/zero/1.0/) applies to the data made available in this article, unless otherwise stated in a credit line to the data.

\section{References}

1. Laux CJ, Grubhofer F, Werner CML, Simmen HP, Osterhoff G. Current concepts in locking plate fixation of proximal humerus fractures. J Orthop Surg Res. 2017;12(1):137. Published 2017 Sep 25. doi:10.1186/s13018-017-0639-3

2. Maier D. Jäger M, Strohm PC. Südkamp NP. Treatment of proximal humeral fractures - a review of current concepts enlightened by basic principles. Acta Chir Orthop Traumatol Cech. 2012;79(4):30716.

3. Jung WB, Moon ES, Kim SK, Kovacevic D, Kim MS. Does medial support decrease major complications of unstable proximal humerus fractures treated with locking plate?. BMC Musculoskelet Disord. 2013;14:102. Published 2013 Mar 22. doi:10.1186/1471-2474-14-102.

4. Sproul RC, lyengar JJ, Devcic Z, et al. A systematic review of locking plate fixation of proximal humerus fractures[J]. Injury. 2011;42(4):408-13.

5. Egol KA, Shulman BS, Belayneh R, Karia RJ, Zuckerman JD.Does a Learning Curve Exist for the Surgical Treatment of Proximal Humerus Fractures?Bull Hosp Jt Dis (2013). 2018 Dec;76(4):265268.PMID:31513512.

6. Wang B, Zhu C, Wang P, Wu ZZ, Zhang QF. Zhongguo Gu Shang. 2018;31(9):794-8. doi:10.3969/j.issn.1003-0034.2018.09.003.

7. Leiblein M, Verboket R, Marzi I, Wagner N, Nau C. Nonunions of the humerus - Treatment concepts and results of the last five years. Chin J Traumatol. 2019;22(4):187-95. doi:10.1016/j.cjtee.2019.04.002.

8. ill H,Hepp P,Korner J,et a1.Dai, Chai J, Wang Y C, et al. Meta- analysis comparing locking plate fixation with hemiarthroplasty for complex proximal humeral fractures[J].Eur J Orthop Surg Traumatol,2014,24(3):305-313.

9. Frima H, Houwert RM, Beks RB, van Heijl M, van der Velde D, Beeres FJP. Proximale humerusfracturen [Proximal humerus fractures; conservative or surgical treatment?]. Ned Tijdschr Geneeskd. 2019;163:D3096. Published 2019 Jan 3. 
10. Wu JW, Shen HL, Liu LM, Gao ZH. Beijing Da Xue Xue Bao Yi Xue Ban. 2016;48(4):683-5.

11. Kralinger F, Unger S, Wambacher M, Smekal V, Schmoelz W. The medial periosteal hinge, a key structure in fractures of the proximal humerus: a biomechanical cadaver study of its mechanical properties. J Bone Joint Surg Br. 2009;91(7):973-6. doi:10.1302/0301-620X.91B7.21857.

12. Hufeland $M$, Hamed $G$, Kubo H, et al. Blood supply in the bicipital groove: A histological analysis. Orthop Rev (Pavia). 2019;11(1):8106. Published 2019 Mar 29. doi:10.4081/or.2019.8106.

13. Park SG, Ko YJ. Medial Buttress Plating for Humerus Fractures With Unstable Medial Column[J]. J Orthop Trauma. 2019;33:e352-9.

14. Theopold J, Marquaß B, Fakler J, Steinke H, Josten C, Hepp P. The bicipital groove as a landmark for reconstruction of complex proximal humeral fractures with hybrid double plate osteosynthesis. BMC Surg. 2016;16:10. Published 2016 Mar 12. doi:10.1186/s12893-016-0125-6.

15. Liu ZD, Ma MT, Chen JH, Fu ZG, Jiang BG. Beijing Da Xue Xue Bao Yi Xue Ban. 2017;49(6):1003-7.

16. Jung WB, Moon ES, Kim SK, Kovacevic D, Kim MS. Does medial support decrease major complications of unstable proximal humerus fractures treated with locking plate?. BMC Musculoskelet Disord. 2013;14:102. Published 2013 Mar 22. doi:10.1186/1471-2474-14-102.

17. Theopold J, Marquaß B, Fakler J, Steinke H, Josten C, Hepp P. The bicipital groove as a landmark for reconstruction of complex proximal humeral fractures with hybrid double plate osteosynthesis. BMC Surg. 2016;16:10. Published 2016 Mar 12. doi:10.1186/s12893-016-0125-6.

18. Kavuri V, Bowden B, Kumar N, Cerynik D. Complications Associated with Locking Plate of Proximal Humerus Fractures. Indian J Orthop. 2018;52(2):108-16. doi:10.4103/ortho.IJOrtho_243_17.

\section{Tables}

Tables 1-5 are not available with this version. 